\title{
Epigenomic plasticity within populations: its evolutionary significance and potential
}

\author{
LJ Johnson and PJ Tricker \\ School of Biological Sciences, University of Reading, Reading, UK
}

\begin{abstract}
Epigenetics has progressed rapidly from an obscure quirk of heredity into a data-heavy 'omic' science. Our understanding of the molecular mechanisms of epigenomic regulation, and the extent of its importance in nature, are far from complete, but in spite of such drawbacks, population-level studies are extremely valuable: epigenomic regulation is involved in several processes central to evolutionary biology including phenotypic plasticity, evolvability and the mediation of intragenomic conflicts. The first studies of epigenomic variation within populations suggest high levels of phenoty-
\end{abstract}

pically relevant variation, with the patterns of epigenetic regulation varying between individuals and genome regions as well as with environment. Epigenetic mechanisms appear to function primarily as genome defences, but result in the maintenance of plasticity together with a degree of buffering of developmental programmes; periodic breakdown of epigenetic buffering could potentially cause variation in rates of phenotypic evolution.

Heredity (2010) 105, 113-121; doi:10.1038/hdy.2010.25 published online 24 March 2010

Keywords: epigenetics; population genomics; canalisation; capacitance

\section{Introduction: the potential and pitfalls of population epigenomics}

Genomics is to genetics as demography is to biography: it is focused not on individual components, but rather on their statistical properties in the aggregate and their interactions and interconnections in context. Population genetics and epigenetics are now, similarly, going -omic, and they are not alone: the secretome, fermentome, junkome, diseasome, and even human speechome have all been suggested as objects of study. Indeed, collecting and classifying jargon terms appended by the -ome suffix is now a tongue-in-cheek scientific field in itself: omeomics, coined by science writer Richard Grant in 2005.

The rapid expansion of the omeome certainly has some amusement value, but it would be wildly inaccurate to dismiss the -omics revolution as simply a jargon spree (Evans, 2000). Unprecedented gluts of data from sequencing and microarray technologies have necessitated new techniques for statistical and computational analysis, and new areas of biological science are developing as previously disparate research fields broaden and merge. One such new field is at the confluence of population genetics, genomics and epigenetics.

The consensus definition of an epigenetic trait obtained at a 2008 Cold Spring Harbor meeting was 'a stably heritable phenotype resulting from changes in a chromosome without alterations in the DNA sequence' (Berger et al., 2009). Epigenetics, by extension, is the study of these traits, their heritability and variation. This definition, which we will use throughout this paper,

Correspondence: Dr LJ Johnson, School of Biological Sciences, University of Reading, Lyle Building, Whiteknights Campus, Reading RG6 6BX, UK. E-mail: l.j.johnson@reading.ac.uk

Received 14 December 2009; revised 27 January 2010; accepted 11 February 2010; published online 24 March 2010 excludes cis-regulatory DNA sequence elements such as transcription factor binding sites.

The extension of epigenetics to epigenomics involves a broadening of focus from specific loci to large or complete sets of epigenetic traits affecting the phenotype of an individual. Similarly, one can broaden focus from a single individual to the cloud of related genomes, and their associated epigenomes, which make up a population or species. Population genomics, by detailing and quantifying diversity within species, not only catalogues variation in economically, medically or agriculturally important genes, but also hints at possible evolutionary trajectories. Population epigenomics (Richards, 2008; Vieira et al., 2009) has similar potential, but unfortunately, also some of the same problems, and those exacerbated.

First, as more and longer genomes become complemented by epigenomes and population epigenomes, we may very shortly find we have more data than sense. When genetic information was hard to obtain, individual sequences or sequence alignments were subject to intense analysis and reanalysis to squeeze understanding out of a few precious data. Today, only a small minority of DNA sequences uploaded to databases will ever be looked at by human eyes, much less examined in detail; instead, they will be summarised in vast statistical analyses. We will soon have a similarly plentiful supply of epigenomic data, but with nothing like the toolkit of analytical techniques, the theoretical background or the molecular understanding, which we have built up over the last three decades for genetics and genomics. To appreciate the gap between the two fields, consider that epigenetics papers in broad-focus journals still begin by defining epigenetics.

A second note of caution should be sounded regarding model systems. When cheap, rapid sequencing became available, the very concept of the model system began to 
seem outmoded. Reports of its demise have been greatly exaggerated, or are, at the very least, premature: although genome sequences are becoming available for increasingly obscure organisms, the bulk of epigenomics research centres on the molecular biology workhorse Arabidopsis, supported by other established model systems such as Drosophila, mice, maize and humans. In population genomics, too, the classic model systems reign: currently, the largest population data sets are for yeasts (Tsai et al., 2008; Liti et al., 2009), and hundreds of Drosophila individuals are in the process of being sequenced (Ledford, 2008). Yet it appears that many traditional model systems are atypical as regards the epigenetic control of their genomes. The very convenience for which laboratory organisms have been adopted makes them unusual in that most have rapid and precocious development, and small genomes with a low complement of repetitive and selfish elements. This suggests that they could be less prone to transcriptional noise (Bird, 1995), and may be likely to need less epigenetic silencing or genome defence. Model organisms have also typically undergone intense artificial selection and population bottlenecks in the process of domestication; these cause inbreeding which may affect epigenetic regulation (Walbot, 2009). Finally, those organisms that have become established as models have clear-cut systems of heredity that lend themselves to straightforward genetic analysis, and can readily be transformed with foreign DNA, which might tend to exclude species with epigenetic genome defence systems (Neurospora crassa being a notable exception (Selker, 1990)).

This may mean that we have unintentionally focused on model systems with an atypically low capability for complex epigenetic control. Drosophila melanogaster shows debatable evidence even of DNA methylation, containing only one recognisable DNA methyltransferase (Marhold et al., 2004) compared with several in its relatives Drosophila pseudoobscura and Anopheles gambiae. Similarly, the budding yeast Saccharomyces cerevisiae has lost its capacity to regulate genes through RNA interference while its relatives Saccharomyces castelli and Candida albicans retain this system (Drinnenberg et al., 2009). Not even the laboratory mouse is above suspicion, as many commonly used strains are the result of hybridisation between several subspecies (Wade et al., 2002), and hybrids too may have atypical epigenomes (Hegarty et al., 2008).

Maize, of course, is studied because of its economic importance rather than genetic tractability, so may provide our best hope of a 'normal' epigenome, although the extremely low population sizes that prevailed during its recent domestication bode less well for its showing typical population genomics (Wang et al., 1999). Indeed, epimutations were first described in maize, in 1956 (Brink, 1956 for a historical perspective, see Chandler and Alleman, 2008). It is perhaps no coincidence that maize was the study system of Barbara McClintock, who mistrusted the molecular genetics of her day and saw her discovery of transposable elements as a glaring counterexample to others' reductionist thinking (Comfort, 1995). To her, epigenetic and positional effects were of such paramount importance that she even avoided the use of the word 'gene,' and aligned herself with Goldschmidt (McClintock, 1951), who argued the gene was analogous to a finger on a violin string, in that its position was more important than its composition (Goldschmidt, 1951).

If genomics technologies adapt readily to new species, the distinction between model and non-model system may indeed fade in the near future. This would be advantageous not just because more data allow for better analyses, but because non-model systems are likely to be more representative of biological normality.

Finally, there is a temptation to be sensationalist regarding new and intriguing findings. Naïve or contrarian interpretations of epigenetic discoveries can raise the spectre of Lamarck, as they allow for the limited inheritance of acquired characteristics, or even that of Lysenko, who believed that environmental manipulations could induce radical and heritable phenotypic change. It is therefore important to stress, first, that many epigenetic responses are determined and controlled by DNA genes, and second and more fundamentally, it makes no difference to the basic mathematics of evolution by natural selection whether or not the heritable changes involved consist solely of alterations in DNA sequence. A full understanding of epigenomics may make important and unexpected contributions to evolutionary and developmental biology, but this will extend, not overthrow, the Darwinian synthesis.

\section{History: plasticity, populations and evolution}

A realistic grasp of the extent and the mechanisms of epigenomic control will be of immense value to population geneticists and evolutionary biologists because it is largely through epigenomic means that organisms achieve phenotypic plasticity: the capacity for a single genotype to result in different phenotypes tailored to the environment. This is observed most clearly in plants, which, for obvious reasons, are more strongly obligated than most animals to make the best of potentially unfavourable local conditions. Debates regarding the ecological importance and evolutionary consequences of plasticity within populations have a long and occasionally acrimonious history (reviewed by Pigliucci et al., 2006), but two necessary prerequisites to adequately assess its importance and effect are only now becoming available: first, an understanding of the molecular basis of that plasticity, and second, sufficient relevant data regarding the epigenetic states of large numbers of genes per individual, replicated within populations. Population epigenomics provides a chance to resurrect these debates and resolve them-or perhaps rethink them.

Phenotypic plasticity has been recognised since the beginnings of quantitative genetics, when variation was first partitioned into genetic and environmental components. Even before Mendelian genetics was understood, natural historians grasped that plastic development, capable of responding to environmental signals, could be a powerful adaptation: JM Baldwin (1896) noted that 'The creatures which can stand the 'storm and stress' of the physical influences of the environment, and of the changes which occur in the environment, by undergoing modifications of their congenital functions or of the structures which they get congenitally-these creatures will live; while those which cannot, will not'.

Over the following 50 years, the triumph of the chromosome theory, and the huge advances in genetics that followed, fostered a less mystical attitude to 
organismal development: although the processes involved were not understood in any detail, they were at least acknowledged as investigable, and tentatively named. When Waddington coined 'epigenetics', his definition encompassed most of what we would now call developmental biology, but he stressed that any phenotype was a product of interaction between the inherent genetics of the organism and the influence of the environment-that is, plasticity was built into this definition. Our definition of epigenetics has since changed, but is still closely related to plasticity, as epigenetics is heavily involved in creating a multipurpose genome, allowing for the same genetic information to yield multiple cell types, highly dissimilar life cycle stages, and the existence of alternative developmental pathways for a single organism based on its own and its ancestors' environment.

The evolutionary origins of epigenomic mechanisms have also been the subject of intriguing debate and speculation, although their original and current functions may be drastically different. DNA methylation, for example, could have arisen as a means to distinguish between the original and newly synthesised strand in mismatch repair-a function it still fulfils today (Modrich, 1989). Remethylation of hemimethylated DNA before the next replication cycle would result in more or less precise heritability of methylation patterns. However, it seems more likely that epigenetic modification evolved to fulfil a function it still holds, as a genome defence against invaders such as viruses or transposable elements (reviewed by Johnson, 2007); bacterial restriction-digestion systems are a well-known example of methylation as genome defence in prokaryotes.

\section{Measuring epigenetic variation in populations}

Of the three major methods of epigenetic regulationmethylation, histone modification and RNA interference-the best whole-genome information currently available pertains to methylation patterns. Even here, our understanding is patchy. Unlike genotyping, which can be performed using almost any tissue sample, epigenotyping should ideally match individuals for tissue type and life-history stage, as tissue types differ drastically in their methylation profiles (Lister et al., 2009) and this variation within the individual organism could be confounded with between-individual variation. There is then a risk that any findings may be relevant only to that specific cellular context, and these difficulties are further compounded by the complex interactions between environment, epigenotype, and also in some cases maternal effects (Kadota et al., 2007). The ENCODE (ENCODE Project Consortium, 2004) and modENCODE (Celniker et al., 2009) projects, which aim to characterise all functional elements in the human genome, and in the fly and worm genomes, respectively, include chromatin structure marks as well as DNA sequence-based functional elements and aim to compile cell- and tissuespecific reference data. These will be of great value in quantifying intra-individual variation, but to disentangle this from inter-individual variation may require new analytical methods, perhaps similar to the systems biology approaches currently being used to elucidate the structure of gene and protein networks (Albert, 2007).
Techniques incorporating genetic and epigenetic analyses are now revealing, for a few species, the extent of existing epigenetic variation, and the types of genes and genome regions in which we might expect phenotypically relevant epigenetic variation. A recent review by Richards (Richards, 2008) establishes that there is significant and function-affecting genetic variability in the genes responsible for epigenetic control. Data are largely confined to mammals and flowering plants, and we will consider these two groups separately, in spite of the similarity of the techniques used. Evolutionary divergence between these groups, whose last common ancestor is estimated to have lived 1.6 billion years ago (Meyerowitz, 2002), far exceeds that within, as humans and mice diverged around 100 million years ago (Bininda-Emonds et al., 2007), and maize and Arabidopsis around 250 million years ago (Brandl et al., 1992). Until the tree of life is better covered, we cannot know which features of plant and mammalian epigenetic systems are independently derived: indeed, such similarities as are observed should perhaps surprise us.

The methylome of Arabidopsis thaliana (Zhang et al., 2006) was the first to be published in 2006, and population variation is also best understood in this species. Interestingly, patterns of DNA methylation vary between genome regions such that particular genome regions are rich in polymorphic sites whereas others, such as those rich in transposable elements, are almost always methylated (Riddle and Richards, 2002).

Arabidopsis studies have also made use of epigenetic inbred lines (Richards, 2009), which maximise epigenetic and minimise genetic variation. These show phenotypic variation for several important traits (Johannes et al., 2009), which is heritable over at least eight generations in a uniform environment, and maintain epi-allelic plasticity (Reinders et al., 2009). Ecotypes of Arabidopsis collected from drastically differing environments have also been compared in several epigenomic studies. As well as DNA methylation differences (Vaughn et al., 2007), ecotypes differ in their short interfering RNA complements (Zhai et al., 2008).

An alternative and equally promising approach, used in both Arabidopsis and maize, has been to use mutants in genes involved in methylation to identify target genes whose expression varies between mutant and wild type. These can then be presumed to be under particularly strong epigenetic control and subjected to further epigenetic and genetic analysis. As expected under this scenario, the expression of genes identified in this way varied drastically between inbred lines of maize (Makarevitch et al., 2007) and epigenetically repressed Arabidopsis transposable elements differed in their activities in methylation mutants (Rangwala and Richards, 2007). Evolutionary studies of these sets of genes would also be valuable.

In mammals, variation in epigenetic states such as methylation, and in level of epigenetic control, also appears to differ in complex ways both between genome regions and between individuals. Genes in areas of low gene density in the human genome show greater environmental sensitivity, and this appears to be because of epigenetic factors such as chromatin structure, which is suggested to be more changeable in gene-sparse regions containing few housekeeping genes (Choi and Kim, 2007). Extending the human epigenome project to a 
population-wide study showed intriguing differences between CpG-rich and CpG-poor regions of the genome: CpG-rich regions have low but consistent methylation levels in most individuals, but the methylated sites are variable. In $\mathrm{CpG}$-poor regions, by contrast, there is more variance in the average proportion of methylated sites, but the precise sites that are methylated are more consistent across individuals (Bock et al., 2008).

As in plants, one tactic used in the search for epigenetic variation is to compare individuals who are as near as possible genetically identical, so that heritable differences are likely because of epigenetic variation. In this vein, monozygotic twin rats can be made by separating a single blastocyst, and have been found to be more similar to one another, for multiple traits, than are genetically identical twin sisters from extremely inbred lines showing no heterozygosity, whose origin is dizygotic (Gartner, 1990). The fact that epigenetic variation is observed even between these individuals, who are closely related by ancestry as well as genetically identical, and share maternal effects as well as environment, suggests we might expect high levels of phenotypically relevant epigenomic variation in mammalian populations.

The second tactic is to use genetically divergent lines, as with ecotypes of Arabidopsis. In this study, epigenetic differences are greater, but more difficult to disentangle from genetic effects. Using mice from divergent selection experiments, Hager et al. (2009) found that epigenetic imprinting effects were small and precise, but common; $30 \%$ of loci with an additive effect on bodyweight showed imprinting.

Human epigenomic studies are hampered by the technical difficulties and ethical problems of sampling diverse human populations systematically in different tissues and life stages. However, both tactics have been used. Identical twins show some epigenetic divergence, which increases with age and differing lifestyles (Fraga et al., 2005), and an analysis of cell lines sampled from diverse individuals showed that chromatin state clusters by family (Kadota et al., 2007). Cancer genetics has also developed an epigenetic wing, as it seems germline epimutations or epigenetic variants may confer increased susceptibility to some cancers (Zingg and Jones, 1997).

\section{Epigenomics and evolution}

In spite of the difficulties and caveats outlined above, to achieve a true understanding of evolutionary epigenomics, it will be vital to consider population level and evolutionary processes. Just like new genomic variation, epigenomic variation must succeed and invade at the population level before it can either contribute to between-species divergence or constitute a significant means of gene regulation. For this reason, although there is no reason novel epimutations should be adaptive, an epigenomic trait that occurs in a whole population in response to a previously encountered environmental condition can generally be expected to have an adaptive value, in the same sense as that in which physiological responses are generally assumed to benefit the organism.

Intergenic and gene promoter epigenetic marks can be re-set and re-localised after meiosis, and these processes are involved in directing post-zygotic development: for example, the epigenetically regulated Flowering Locus C determines flowering time in winter-annual Arabidopsis from high latitudes (Choi et al., 2009) in addition to its involvement in chromatin silencing elsewhere in the genome (Baurle et al., 2007). Epigenetic control, paradoxically, can also function as a noise-generating system, which can contribute to developmental programs by allowing heterogeneity in genetically identical cells (Hemberger et al., 2009) and breaking symmetries when needed (Gordon et al., 2009).

Adaptive epigenetic mechanisms can have detrimental side effects. Repeat-counting mechanisms that may have evolved to monitor copy number of genomic parasites (Chandler and Alleman, 2008) also trigger the epigenetic regulation of copy number variation in endogenous genes (Riddle and Richards, 2002). Epigenetics, therefore, also influences evolution by restricting the range of possible gene duplications-the most dramatic example being repeat-induced point mutation, which has effectively halted gene duplication in Neurospora crassa (Galagan et al., 2003).

Developmental and regulatory plasticity itself offers obvious advantages at an individual level, as Baldwin saw (1896, quoted above). However, the advantages and drawbacks of plasticity at the level of the population, or of the evolutionary lineage, have been more hotly contested and are more poorly understood.

Complicating matters, genotype and epigenotype can be tightly or loosely correlated, and epigenotype at one locus can often be determined by genotype at another. The generation and transmission dynamics of epigenetic variants may also differ greatly from those of conventional DNA genes, which show low constant mutation rates and Mendelian inheritance. Fortunately, population geneticists already deal with some such quirky behaviour in the context of mobile elements and intragenomic conflicts (Burt and Trivers, 2006).

In this study, we will cover three areas in which epigenomics will hold particular interest for population and evolutionary geneticists: the effects of epigenomic variability on evolvability; the intriguing possibility of punctuational 'genome shock' caused either by hybridisation or breakdown of epigenomic control; and the possibilities for evolutionary conflict either within the epigenome, or between the genome and epigenome.

\section{Plasticity and evolvability}

One of the most intriguing aspects of epigenetic discoveries is the potential effect of plastic epigenetic control systems on the evolvability of phenotypic traits, and therefore of species and diversity. The extent and type of epigenetic variation determines how a trait will evolve (Wolf et al., 2009). Generally, plasticity has been understood to facilitate phenotypic evolution, first by the simple means of allowing populations to survive long enough to adapt (the Baldwin effect (Crispo, 2007)), and second by canalisation, in which facultative plastic responses become obligate stages of development. Canalisation does not necessarily require any epigenetics in the modern sense, and could realistically be achieved solely through changes at the DNA sequence level, such as, for example, an alteration to a promoter sequence causing the constitutive expression of a previously developmentally regulated protein. We would suggest, however, that epigenetic regulation is likely to become 
involved in this kind of adaptation because it allows for an intermediate level of control, providing stable repression through replication cycles and, potentially, the gradual assumption of a plastic response into the co-ordinated higher-order DNA replication structure (reviewed in Corpet and Almouzni, 2009).

The extra layer of regulatory control provided by epigenomic regulation also adds more axes to the adaptive landscape, which can smooth craggy adaptive landscapes and facilitate the evolution of complex phenotypes (Arnold et al., 2001).

However, plasticity may have counteracting negative effects on evolvability (Price et al., 2003); for instance, it can reduce the correlation between an individual's genotype and its fitness, weakening the effect of natural selection. Whitlock (1996) draws a parallel between adaptive plasticity and the concept of niche breadth in ecology: plasticity allows for a broader niche, so response to selection is slower and genetic load higher.

The heritable nature of epigenomic control differentiates it from other plasticity mechanisms. There is evidence from wild plant populations that transgenerational plasticity is adaptive and leads to increased fitness (Galloway and Etterson, 2007) and from microorganism populations that 'environmental anticipation has been selected for during evolution' (Mitchell et al., 2009). Transgenerational inheritance of epigenetic marks increases phenotypic variation in a manner that appears to condition the organism for stabilising selection (Rando and Verstrepen, 2007).

The longevity of epigenetic memory can also vary and evolve. Theoretical work suggests that a long epigenetic memory will increase optimal plasticity, and the optimal length of epigenetic memory depends on existing adaptation (Pal, 1998). Dual inheritance of genetic and epigenetic information is particularly favourable if the population is far from as adaptive peak. Importantly, plasticity combined with memory allows for the maintenance of high levels of genetic variation although a population is close to phenotypic optimum. This last gives solid theoretical support to the previously somewhat nebulous concept of 'evolutionary capacitance': the idea that silent genetic variation can build up over generations, to be expressed or released periodically.

\section{Capacitance and genome shock}

\section{Concepts of capacitance}

The conditions that are postulated to trigger a release from evolutionary capacitance, and reveal unexpected variability in a population, are not subtle shifts in selective pressures but drastic events with genome-wide consequences. These include prion accumulation, selfish element invasion, hybridisation, inbreeding or physiological stresses brought about by extreme environmental change. 'Omics' approaches are therefore particularly appropriate for investigating capacitance.

Heat shock proteins, and other molecular chaperones, are potential mediators of evolutionary capacitance. By allowing a range of mutant proteins to fold normally, they can mask variation which is revealed in mutants. Disruption of the HSP90 heat-shock protein, which acts as a molecular chaperone for over 100 signalling proteins, increases variability (Rutherford and Lindquist, 1998) and has been shown to leave a functional, heritable epigenetic 'mark' in isogenic Drosophila melanogaster (Sollars et al., 2003). At the whole genome or population level, although, not all of these responses have a consistent epigenetic basis, and recent work (Specchia et al., 2010) suggests an alternative explanation for increased variability, namely that mutation of HSP90 frees transposons from suppression and so increases insertional mutation.

Heat shock proteins have a known role in facilitating the folding of non-mutant proteins; as with the yeast prion previously proclaimed as an evolvability switch (Partridge and Barton, 2000), there is as yet no evidence that heat shock mutations are ever adaptive in the wild, let alone that their role in periodically revealing genetic variation can truly be regarded as a function.

The bistable, periodic nature of capacitance and release irresistibly calls to mind the theories of punctuated equilibrium first developed and espoused by palaeontologists (Gould and Eldredge, 1977), and supported by some recent work in molecular phylogenetics (Pagel et al., 2006). Many researchers have indeed made this connection, suggesting that adaptive spurts are caused by physiological stress disrupting epigenetic variation. Some add extra accelerants to these evolutionary explosions, pointing out that gene conversion or recombination can be accelerated by changes in chromatin conformation (Cummings et al., 2007) or that transposable elements, freed from the restraint of epigenomic genome defences, could quickly expand and restructure the genome (Zeh et al., 2009). Hybridisation may trigger these processes, as hybrids show extensive epigenetic dysfunction (reviewed by Michalak, 2008).

Polyploidy is another potential trigger for epigenetic revolution. The advantages of changes in gene expression (Ni et al., 2009) and restructuring in hybrids (Pennington et al., 2008) and stable allopolyploid plants, Leitch and Leitch (2008) argue, are responsible for much of their diversity and success. If epigenomic cryptic variation is revealed in response to shock or challenge, it should lead to rapid adaptation and increased population evolvability (sensu Hansen (2006)). Rapid evolution of novel allopolyploids has been shown in several species, including fireweeds in which rapid changes result from population-level interactions with diploid parental species (Husband and Sabara, 2004). Buggs et al. (2009) have also shown that in salsify (Tragopon) allopolyploids, the silencing of homeologous chromosomes varies between different populations. Rapid divergence is observed in expression pattern between gene duplicates, particularly under stress (Liu and Adams, 2007). The broad synthesis emerging from large molecular data sets is that these genomic processes are, in large part, epigenetically directed (reviewed by Madlung and Comai, 2004).

\section{Conflicts and antagonistic coevolution}

Epigenetic regulation also strongly influences population-level processes by mediating genetic conflicts, in particular that between the endogenous nuclear genome and selfish mobile elements such as transposons, retroviruses and retrotransposons. Another review in this issue deals with the epigenomics of transposable element 
control, so here it will be sufficient to state that the intensity of conflicts varies across genomes, populations and environments, again favouring an -omics approach to the study of these global, omic-level controls, which give the individual an advantage over its genomic parasites, toward whom the battle is otherwise unfairly skewed. Epigenetic regulation of transposable elements can be position dependent or otherwise nonuniformly distributed across the genome-for example, transposons within a heterochromatic domain can be silenced en masse (Grewal and Moazed, 2003)_and in addition can depend on environmental conditions, such as temperature in the case of Drosophila P elements (Vieira et al., 2009). Some transposable elements are also targeted to particular chromatin forms-in the 'ecology of the

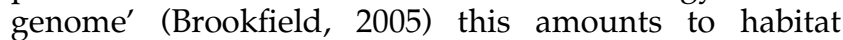
specialisation-and cell type and developmental stage also has a role. In animal somatic cells, or plant cells irreversibly committed to vegetative growth, for example, there is no conflict over somatic suppression.

The role of imprinting in intersexual conflict over offspring provisioning is a famous example of epigenetic conflict, and inherited adaptive plasticity results in parent-offspring conflict too. To prevent such conflict, maternal genomes can be actively demethylated in the zygote, effectively 'disarming' sperm (Bird, 2002).

Intrasexual conflicts also involve epigenetics. Dietderived and condition-dependent sexual signals, which are by definition epigenetic sensu Waddington, seem also to be epigenetic in the molecular, non-DNA modification sense.

It is of course important to remember that a single epigene-a locus or other inherited factor whose phenotypic effect is not associated with DNA sequence variation-can evolve more than one function, and can hold those functions sequentially or simultaneously. PEG10 is a transposon-derived placental gene: its orthologue in wallabies is the first imprinting-associated differently methylated region found in marsupials, but there is no orthologue in the platypus (Suzuki et al., 2007); this indicates that the gene originated, probably through transposition, and acquired its imprinted status before the marsupial-placental spilt, but acquired its function in the placenta more recently. In an interesting convergence, imprinting appears to have been similarly co-opted from transposon silencing in plants (Gehring et al., 2009). Transposon-induced epigenetic changes have also been instrumental in causing evolutionary change in plant breeding systems (Martin et al., 2009).

\section{Distinctions}

Pedantic although it may seem, it is important to draw a strict distinction between a system that incidentally increases evolvability but is maintained by selection for other reasons, and an 'evolvability mechanism' sensu stricto. Evolvability can indeed evolve under fluctuating selection (Draghi and Wagner, 2009), but increased evolvability could also be simply a by-product of plasticity-and the fact that this increased evolvability has had important consequences, or even been vital to the evolution of life's current diversity, is irrelevant to the question of whether the evolvability itself is an adaptation. Two parallels may be illustrative. Mutation is essential for evolution and mutation rates are under some degree of genetic control, yet mutation is not an adaptation, as all available evidence supported by theoretical work shows that low mutation rates are almost always favoured in sexual populations; actual mutation rates are likely to be a trade-off between accuracy and energetic expenditure without evolvability being a factor (Sniegowski et al., 2000). Sex, on the other hand, truly is best explained as an evolvability mechanism according to our current understanding: the 'Red Queen' theory, plus that of Weismann (Burt, 2000) and those based on Hill-Robertson effects (Barton and Otto, 2005) are variations on this theme.

For the reasons above-the smoothing of adaptive landscapes and the maintenance of genetic variationwe take the view that epigenetic variation very likely does make species more evolvable and contributes to the evolutionary success of some lineages. Taken together, studies to date strongly suggest that epigenomic responses are canalisation factors. They maintain cryptic variation in the epigenome, reducing direct genome effects while enabling phenotypic plasticity. To resolve whether this evolvability is an adaptation, however, we need more theoretical and practical understanding of the molecular basis and the short- and long-term costs of epigenomic plasticity and a quantitative measure of the extent to which this plasticity aids evolution (such as those being calculated for the benefits of recombination (Agrawal et al., 2005)), plus observational evidence such as the long-term maintenance of a gene with no other function than increasing evolvability. Relevant theoretical calculations of this nature have been attempted with regards to the evolutionary capacitance theory of the maintenance of psi prion of yeast which, assuming large population sizes, low mutation rates and a low cost of maintenance, must provide a new adaptive change every million years to survive mutational degeneration (Masel and Bergman, 2003). Similar work on the more complex plasticity of animals and plants would be extremely valuable.

We should also consider the contribution of transposable element population dynamics affecting the evolvability of the lineages they infest: what we think of as evidence of adaptation at or above the level of the individual may be the result of selfish activity at a lower level.

\section{Summary and pressing questions}

Patterns of epigenetic regulation vary between individuals, and across populations-including populations of transposable elements (Rangwala et al., 2006)-between genome regions, over time, and with environmental stresses both biotic and abiotic (Molinier et al., 2006), and there are complex interactions between the above factors.

Epigenetic mechanisms seem to function primarily as genome defences, but also adaptively or otherwise result in the maintenance of plasticity together with a degree of buffering of developmental programmes (Parkinson et al., 2007). Although epigenetic silencing could potentially be an all-purpose control mechanism-like transcription factors, it is most heavily used in certain areas of genome function, such as defence, noise reduction, mediation of genetic conflicts and the facilitation of plastic responses to environment, which require rapid but sustainable responses. 
In population genetics, a population has a diversity, number of genotypes, variance in fitness, effective population size and other emergent, collective properties. In the same way, whole epigenomes and population data sets have properties an individual epigenotype at a single locus does not. We need new measures of epigenomic influence on these population-level, rather than individual, characteristics, and an extension of statistical data analysis techniques to deal with them. As an example of the advantages of 'omic' analysis, recall that, using gene-level sequence-based methods, proving the effects of natural selection at any one locus or site was extremely difficult: today, the percentage of sites under natural selection can be estimated using wholegenome data (Eyre-Walker, 2006).

Particularly interesting questions for which data will soon be available are, first, are highly epigenetically regulated genes fast- or slow-evolving? Drosophila polycomb response elements are surprisingly fast-evolving (Hauenschild et al., 2008), and silencing proteins also diverge rapidly (Fabre et al., 2005), which could be suggestive of antagonistic coevolution caused by intragenomic or epigenomic conflict.

Second, as data from more species become available, the effect of ecology can be examined. Are plants with high dispersal more plastic, with more variable epigenomes, or more of the genome under some form of epigenomic control? Is this true of generalists, weeds and invasive species?

Third, comparative and evolutionary analyses, particularly those involving intragenomic populations of transposable elements, can shed light on the timescales of epigenetic and genetic adaptation, and determine which is responsible for short- and long-term phenotypic change. This will also clarify the question of the frequency and intensity of 'genome shocks': periods of instability resulting in rapid, drastic restructuring.

Theoretical understanding will be as important as molecular. We might ask: are epigenetic networks wired up to 'help' or 'work with' selection at the genetic level? What are the limits of plasticity (Whitlock, 1996), and are these ecologically imposed or molecular constraints? We also need to combine an understanding of plasticity into other areas of evolutionary biology, as has to some extent been achieved in the area of the evolution of sex and recombination rates (Agrawal et al., 2005). Quantitative and population genetics are largely based on populationlevel phenomena, such as heritabilities and responses to selection, that depend on the way in which variability is generated; it remains to be observed whether the population-level implications of epigenomic modifications will necessitate a mere tweak, or a wholesale rethink, of the fundamental concepts of evolutionary biology.

\section{Conflict of interest}

The authors declare no conflict of interest.

\section{Acknowledgements}

PJT's work is supported by Cocoa Research (UK) Ltd and the Dutch Ministry of Agriculture, Nature and Food Quality (Ministerie van LNV). LJJ is supported by an RCUK Academic Fellowship.

\section{References}

Agrawal AF, Hadany L, Otto SP (2005). The evolution of plastic recombination. Genetics 171: 803-812.

Albert R (2007). Network inference, analysis, and modeling in systems biology. Plant Cell 19: 3327-3338.

Arnold SJ, Pfrender E, Jones AG (2001). The adaptive landscape as a conceptual bridge between micro and macroevolution. Genetica 112-113: 9-32.

Baldwin JM (1896). A new factor in evolution. Am Nat 30: 441-451.

Barton NH, Otto SP (2005). Evolution of recombination due to random drift. Genetics 169: 2353-2370.

Baurle I, Smith L, Baulcombe DC, Dean C (2007). Widespread role for the flowering-time regulators FCA and FPA in RNAmediated chromatin silencing. Science 318: 109-112.

Berger SL, Kouzarides T, Shiekhattar R, Shilatifard A (2009). An operational definition of epigenetics. Genes Dev 23: 781-783.

Bininda-Emonds ORP, Cardillo M, Jones KE, MacPhee RDE, Beck RMD, Grenyer R et al. (2007). The delayed rise of present-day mammals. Nature 446: 507-511.

Bird AP (1995). Gene number, noise reduction and biological complexity. Trends Genet 11: 94-100.

Bird AP (2002). DNA methylation patterns and epigenetic memory. Genes Dev 16: 6-21.

Bock C, Walter J, Paulsen M, Lengauer T (2008). Inter-individual variation of DNA methylation and its implications for largescale epigenome mapping. Nuc Acids Res 35: e55.

Brandl R, Mann W, Sprinzl M (1992). Estimation of the monocot-dicot age through tRNA sequences from the chloroplast. Proc R Soc Lond Ser B-Biol Sci 249: 13-17.

Brink RA (1956). A genetic change associated with the $R$ locus in maize which is directed and potentially reversible. Genetics 41: 872-890.

Brookfield JFY (2005). The ecology of the genome: mobile DNA elements and their hosts. Nat Rev Genet 6: 128-136.

Buggs RJA, Doust AN, Tate JA, Koh J, Soltis K, Feltus FA et al. (2009). Gene loss and silencing in Tragopogon miscellus (Asteraceae): comparison of natural and synthetic allotetraploids. Heredity 103: 73-81.

Burt A (2000). Perspective: sex, recombination, and the efficacy of selection-was Weismann right? Evolution 54: 337-351.

Burt A, Trivers R (2006). Genes in Conflict. Harvard University Press: London.

Celniker SE, Dillon LAL, Gerstein MB, Gunsalus KC, Henikoff S, Karpen GH et al. (2009). Unlocking the secrets of the genome. Nature 459: 927-930.

Chandler V, Alleman M (2008). Paramutation: epigenetic instructions passed across generations. Genetics 178: 1839-1844.

Choi J, Hyun Y, Kang M-J, In Yun H, Yun J-Y, Lister C et al. (2009). Resetting and regulation of FLOWERING LOCUS C expression during Arabidopsis reproductive development. Plant J 57: 918-931.

Choi JK, Kim SC (2007). Environmental effects on gene expression phenotype have regional biases in the human genome. Genetics 175: 1607-1613.

Comfort NC (1995). Two genes, no enzyme: a second look at Barbara McClintock and the 1951 Cold Spring Harbor Symposium. Genetics 140: 1161-1166.

Corpet A, Almouzni G (2009). Making copies of chromatin: the challenge of nucleosomal organization and epigenetic information. Trends Cell Biol 19: 29-41.

Crispo E (2007). The Baldwin effect and genetic assimilation: revisiting two mechanisms of evolutionary change mediated by phenotypic plasticity. Evolution 61: 2469-2479.

Cummings WJ, Bednarski DW, Maizels N (2007). Genetic variation stimulated by epigenetic modification. PLoS One 3: e4075.

Draghi J, Wagner GP (2009). The evolutionary dynamics of evolvability in a gene network model. J Evol Biol 22: 599-611. 
Drinnenberg IA, Weinberg DE, Xie KT, Mower JP, Wolfe KH, Fink GR et al. (2009). RNAi in budding yeast. Science 326: 544-550.

ENCODE Project Consortium (2004). The ENCODE (ENCyclopedia of DNA elements) project. Science 306: 636-640.

Evans GA (2000). Designer science and the 'omics' revolution. Nat Biotechnol 18: 127.

Eyre-Walker A (2006). The genomic rate of adaptive evolution. Trends Ecol Evol 21: 569-575.

Fabre E, Muller H, Therizols P, Lafontaine I, Dujon B, Fairhead $C$ (2005). Comparative genomics in hemiascomycete yeasts: evolution of sex, silencing, and subtelomeres. Mol Biol Evol 22: 856-873.

Fraga MF, Ballestar E, Paz MF, Ropero S, Setien F, Ballestar ML et al. (2005). Epigenetic differences arise during the lifetime of monozygotic twins. Proc Natl Acad Sci USA 102: 10604-10609.

Galagan JA, Calvo SE, Borkovich KA, Selker EU, Read ND, Jaffe $\mathrm{D}$ et al. (2003). The genome sequence of the filamentous fungus Neurospora crassa. Nature 422: 859-868.

Galloway LF, Etterson JR (2007). Transgenerational plasticity is adaptive in the wild. Science 318: 1134-1136.

Gartner K (1990). A third component causing random variability beside environment and genotype. A reason for the limited success of a 30 year long effort to standardize laboratory animals? Lab Anim 24: 71-77.

Gehring M, Bubb KL, Henikoff S (2009). Extensive demethylation of repetitive elements during seed development underlies gene imprinting. Science 324: 1447-1451.

Goldschmidt R (1951). Chromosomes and genes Cold Spring Harb Symp Quant 16: 1-11.

Gordon AJE, Halliday JA, Blankschien MD, Burns PA, Yatagai F, Herman C (2009). Transcriptional infidelity promotes heritable phenotypic change in a bistable gene network. PLoS Biol 7: e1000044.

Gould SJ, Eldredge N (1977). Punctuated equilibria: the tempo and mode of evolution reconsidered. Paleobiology 3 : 115-151.

Grewal SIS, Moazed D (2003). Heterochromatin and epigenetic control of gene expression. Science 301: 798-802.

Hager R, Cheverud JM, Wolf JB (2009). Change in maternal environment induced by cross-fostering alters genetic and epigenetic effects on complex traits in mice. Proc $R$ Soc Lond Ser B-Biol Sci 276: 2949-2954.

Hansen TF (2006). The evolution of genetic architecture. Annu Rev Ecol Evol Systemat 37: 123-157.

Hauenschild A, Ringrose L, Altmutter C, Paro R, Rehmsmeier M (2008). Evolutionary plasticity of polycomb/trithorax response elements in drosophila species. PLoS Biol 6: e261.

Hegarty MJ, Barker GL, Brennan AC, Edwards KJ, Abbott RJ, Hiscock SJ (2008). Changes to gene expression associated with hybrid speciation in plants: further insights from transcriptomic studies in Senecio. Phil Trans $R$ Soc Lond $B$ 363: 3055-3069.

Hemberger M, Dean W, Reik W (2009). Epigenetic dynamics of stem cells and cell lineage commitment: digging Waddington's canal. Nat Rev Mol Cell Biol 10: 526.

Husband BC, Sabara HA (2004). Reproductive isolation between autotetraploids and their diploid progenitors in fireweed, Chamerion angustifolium (Onagraceae). New Phytologist 161: 703-713.

Johannes F, Porcher E, Teixeira FK, Saliba-Colombani V, Simon M, Agier N et al. (2009). Assessing the impact of transgenerational epigenetic variation on complex traits. PLoS Gen 5: e1000530.

Johnson LJ (2007). The genome strikes back: the evolutionary importance of defence against mobile elements. Evol Biol 34 121-129.

Kadota M, Yang HH, Hu N, Wang C, Hu Y, Taylor PR et al. (2007). Allele-specific chromatin immunoprecipitation stu- dies show genetic influence on chromatin state in human genome. PLoS Gen 3: e81.

Ledford H (2008). Population genomics for fruitflies. Nature 453: 1154-1155.

Leitch AR, Leitch IJ (2008). Genomic plasticity and the diversity of polyploid plants. Science 320: 481-483.

Lister R, Pelizzola M, Dowen RH, Hawkins RD, Hon G, TontiFilippini J et al. (2009). Human DNA methylomes at base resolution show widespread epigenomic differences. Nature 462: 315-322.

Liti G, Carter DM, Moses AM, Warringer J, Parts L, James SA et al. (2009). Population genomics of domestic and wild yeasts. Nature 458: 337-341.

Liu Z, Adams KL (2007). Expression partitioning between genes duplicated by polyploidy under abiotic stress and during organ development. Curr Biol 17: 1669-1674.

Madlung A, Comai L (2004). The effect of stress on genome regulation and structure. Ann Bot 94: 481-495.

Makarevitch I, Stupar RM, Iniguez AL, Haun WJ, Barbazuk WB, Kaeppler SM et al. (2007). Natural variation for alleles under epigenetic control by the maize chromomethylase Zmet2. Genetics 177: 749-760.

Marhold J, Rothe N, Mund C, Kuehle K, Brueckner B, Lyko F (2004). Conservation of DNA methylation in dipteran insects. Insect Mol Biol 13: 117-123.

Martin A, Troadec C, Boualem A, Rajab M, Fernandez R, Morin $\mathrm{H}$ et al. (2009). A transposon-induced epigenetic change leads to sex determination in melon. Nature 461: 1135-1139.

Masel J, Bergman A (2003). The evolution of the evolvability properties of the yeast prion [PSI+]. Evolution 57: 1498-1512.

McClintock B (1951). Chromosome organization and genic expression. Cold spring harbor symp. Quant Biol 16: 13-47.

Meyerowitz EM (2002). Plants compared to animals: the broadest comparative study of development. Science 295: $1482-1485$.

Michalak P (2008). Epigenetic, transposon and small RNA determinants of hybrid dysfunctions. Heredity 102: 45-50.

Mitchell A, Romano GH, Groisman B, Yona A, Dekel E, Kupiec $M$ et al. (2009). Adaptive prediction of environmental changes by microorganisms. Nature 460: 220-224.

Modrich P (1989). Methyl-directed DNA mismatch correction. J Biol Chem 264: 6597-6600.

Molinier J, Ries G, Zipfel C, Hohn B (2006). Transgeneration memory of stress in plants. Nature 442: 1046-1049.

Ni Z, Kim E-D, Ha M, Lackey E, Liu J, Zhang Y et al. (2009). Altered circadian rhythms regulate growth vigour in hybrids and allopolyploids. Nature 457: 327-331.

Pagel M, Venditti C, Meade A (2006). Large punctuational contribution of speciation to evolutionary divergence at the molecular level. Science 314: 119-121.

Pal C (1998). Plasticity, memory and the adaptive landscape of the genotype. Proc R Soc Lond Ser B-Biol Sci 265: 1319-1323.

Parkinson SE, Gross SM, Hollick JB (2007). Maize sex determination and abaxial leaf fates are canalized by a factor that maintains repressed epigenetic states. Dev Biol 308: 462-473.

Partridge L, Barton NH (2000). Evolving evolvability. Nature 407: 457-458.

Pennington PD, Costa LM, Gutierrez-Marcos JF, Greenland AJ, Dickinson HG (2008). When genomes collide: aberrant seed development following maize interploidy crosses. Ann Bot 101: 833-843.

Pigliucci M, Murren CJ, Schlichtling CD (2006). Phenotypic plasticity and evolution by genetic assimilation. J Exp Biol 209: 2362-2367.

Price TD, Qvarnstrom A, Irwin DE (2003). The role of phenotypic plasticity in driving genetic evolution. Proc $R$ Soc Lond Ser B-Biol Sci 270: 1433-1440.

Rando OJ, Verstrepen KJ (2007). Timescales of genetic and epigenetic inheritance. Cell 128: 655-668. 
Rangwala SH, Elumalai R, Vanier C, Ozkan H, Galbraith D, Richards EJ (2006). meiotically stable natural epialleles of sadhu, a novel Arabidopsis retroposon. PLoS Gen 2: e36.

Rangwala SH, Richards EJ (2007). Differential epigenetic regulation within an Arabidopsis retroposon family. Genetics 176: $151-160$.

Reinders J, Wulff BBH, Mirouze M, Marí-Ordóñez A, Dapp Ml, Rozhon W et al. (2009). Compromised stability of DNA methylation and transposon immobilization in mosaic Arabidopsis epigenomes. Genes Dev 23: 939-950.

Richards EJ (2008). Population epigenetics. Curr Opinion Gen Develop 18: 221-226.

Richards EJ (2009). Quantitative epigenetics: DNA sequence variation need not apply. Genes Dev 23: 1601-1605.

Riddle N, Richards EJ (2002). The control of natural variation in cytosine methylation in Arabidopsis. Genetics 162: 355-363.

Rutherford SL, Lindquist S (1998). Hsp90 as a capacitor for morphological evolution. Nature 396: 336-342.

Selker EU (1990). Premeiotic instability of repeated sequences in Neurospora crassa. Annu Rev Genet 24: 579-613.

Sniegowski PD, Gerrish PJ, Johnson T, Shaver A (2000). The evolution of mutation rates: separating causes from consequences. Bioessays 22: 1057-1066.

Sollars V, Lu X, Xiao L, Wang X, Garfinkel MD, Ruden DM (2003). Evidence for an epigenetic mechanism by which Hsp90 acts as a capacitor for morphological evolution. Nat Genet 33: 70-74.

Specchia V, Piacentini L, Tritto P, Fanti L, D'Alessandro R, Palumbo G et al. (2010). Hsp90 prevents phenotypic variation by suppressing the mutagenic activity of transposons. Nature 463: 662-665.

Suzuki S, Ono R, Narita T, Pask AJ, Shaw G, Wang C et al. (2007). Retrotransposon silencing by DNA methylation can drive mammalian genomic imprinting. PLoS Genet 3: e55.
Tsai IJ, Bensasson D, Burt A, Koufopanou V (2008). Population genomics of the wild yeast Saccharomyces paradoxus: quantifying the life cycle. Proc Natl Acad Sci USA 105: 4957-4962.

Vaughn MW, Tanurd Ic M, Lippman Z, Jiang H, Carrasquillo R, Rabinowicz PD et al. (2007). Epigenetic natural variation in Arabidopsis thaliana. PLoS Biol 5: e174.

Vieira C, Fablet M, Lerat E (2009). Infra- and transspecific clues to understanding the dynamics of transposable elements. Genome Dynamics Stability 4: 21-430.

Wade CM, Kulbokas EJ, Kirby AW, Zody MC, Mullikin JC, Lander ES et al. (2002). The mosaic structure of variation in the laboratory mouse genome. Nature 420: 574-578.

Walbot V (2009). 10 reasons to be tantalized by the B73 maize genome. PLoS Genet 5: e1000723.

Wang R-L, Stec A, Hey J, Lukens L, Doebley J (1999). The limits of selection during maize domestication. Nature 398: 236-239.

Whitlock MC (1996). The red queen beats the jack-of-all-trades: the limitations on the evolution of phenotypic plasticity and niche breadth. Am Nat 148: S65-S77.

Wolf JB, Frankino WA, Agrawal AF, Brodie Iii ED, Moore AJ (2009). Developmental interactions and the constituents of quantitative variation. Evolution 55: 232-245.

Zeh DW, Zeh JA, Ishida Y (2009). Transposable elements and an epigenetic basis for punctuated equilibria. Bioessays 31: 715-726.

Zhai J, Liu J, Liu B, Li P, Meyers BC, Chen X et al. (2008). Small RNA-directed epigenetic natural variation in Arabidopsis thaliana. PLoS Genet 4: e1000056.

Zhang X, Yazaki J, Sundaresan A, Cokus S, Chan SW, Chen H et al. (2006). Genome-wide high-resolution mapping and functional analysis of DNA methylation in Arabidopsis. Cell 1126: 1189-1201.

Zingg J-M, Jones PA (1997). Genetic and epigenetic aspects of DNA methylation on genome expression, evolution, mutation and carcinogenesis. Carcinogenesis 18: 869-882. 